\title{
Efficacy of Scorpion Antivenom in Grade III and IV Scorpion Envenomation
}

\author{
Rajniti Prasad ${ }^{1} \cdot$ Abhishek Kumar $^{1} \cdot$ Om Prakash Mishra ${ }^{1} \cdot$ Tej Bali Singh ${ }^{2} \cdot$ Ankur Singh $^{1}$
}

Received: 9 December 2017 / Accepted: 17 April 2018 / Published online: 27 April 2018

(C) Dr. K C Chaudhuri Foundation 2018

To the Editor: Scorpion envenomation in children is a life threatening emergency. Most of the children can die with severe scorpion envenomation if untreated. It is relatively less severe condition in adults. Scorpion antivenom (SAV) was available in few countries. Now with the free availability of antivenom, more rational treatment is use of SAV to neutralize the venom present in the circulation and body fluids. There are limited studies in pediatric age group assessing role of SAV in scorpion envenomation [1]. Hence this study was planned to assess the efficacy of SAV in pediatric age group.

This study was conducted in Department of Pediatrics, Institute of Medical Sciences, Banaras Hindu University, Varanasi. The period of study extended from March 2014 through December 2016. Ethical clearance was taken from Institute Ethical Committee. The study included children with history of scorpion envenomation who were admitted in our pediatric ward/ Pediatric intensive care unit with following inclusion criteria: Patients of both sexes, in the age range of 1-14 y, presenting to the emergency within $48 \mathrm{~h}$ of red scorpion (Mesobuthus tumulus) sting and associated with clinical manifestations of scorpion envenomation with grade III and IV. Patients in group A received scorpion antivenom plus prazosin $(n=32)$ as per protocol. Patients in group B received prazosin $(n=34)$ only. The present study included 66 patients with 45 boys. Mean age of presentation in group A and group $\mathrm{B}$ was $5.54 \pm 3.2 \mathrm{y}$ and $6.28 \pm 3.93$ y respectively. Fourteen out of 32 children of group A, had clinically improved within $12 \mathrm{~h}$ of hospitalization against none in control group which was a significant finding ( $p$ 0.001). Twenty seven patients

Ankur Singh

pediaankur@gmail.com

1 Department of Pediatrics, Institute of Medical Sciences, Banaras Hindu University, Varanasi, Uttar Pradesh, India

2 Division of Biostatistics, Department of Preventative and Social Medicine, Institute of Medical Sciences, Banaras Hindu University, Varanasi, Uttar Pradesh, India
(84.37\%) of group A recovered within $24 \mathrm{~h}$ compared to only 15 patients $(44.11 \%)$ of group B. The mean duration of hospitalization in group A $(62.91 \pm 26.09 \mathrm{~h})$ was significantly lower than mean duration of hospitalization in group $\mathrm{B}$ $(80.12 \pm 18.01 \mathrm{~h})$.

Many scientists have demonstrated the benefit of use of scorpion antivenom in their studies [2-5].

Scorpion envenomation in children is a life-threatening event. Prazosin and supportive treatment has improved the outcome in pediatric age group. The role of scorpion antivenom has been less studied in pediatric population. Our study points towards the beneficial role of antivenom in the pediatric age group. But, benefit is species specific; the role of antivenom in other species' envenomation needs to be further studied.

\section{Compliance with Ethical Standards}

Conflict of Interest None.

\section{References}

1. Pandi K, Krishnamurthy S, Srinivasaraghavan R, Mahadevan S. Efficacy of scorpion antivenom plus prazosin versus prazosin alone for Mesobuthus tamulus scorpion sting envenomation in children: a randomised controlled trial. Arch Dis Child. 2014;99:575-80.

2. Bawaskar HS, Bawaskar PH. Efficacy and safety of scorpion antivenom plus prazosin compared with prazosin alone for venomous scorpion (Mesobuthus tamulus) sting: randomised open label clinical trial. BMJ. 2011;342:c7136.

3. Natu VS, Murthy RK, Deodhar KP. Efficacy of species specific antiscorpion venom serum (AScVS) against severe, serious scorpion stings (Mesobuthus tamulus concanesis Pocock)-an experience from rural hospital in western Maharashtra. J Assoc Physicians India. 2006;54:283-7.

4. Boyer LV, Theodorou AA, Berg RA, et al. Antivenom for critically ill children with neurotoxicity from scorpion stings. N Engl J Med. 2009;360:2090-8.

5. Prasad R, Mishra OP, Pandey N, Singh TB. Scorpion sting envenomation in children: factors affecting the outcome. Indian J Pediatr. 2011;78:544-8. 